\title{
An Intelligent Evaluation Algorithm for Operator Training
}

\author{
Jisheng Zhang ${ }^{\mathrm{a}}$, Kanheng Zhou ${ }^{\mathrm{b}}$, Jianrong Zheng ${ }^{\mathrm{c}}$ \\ School of Mechanical and Power Engineering, East China University of Science and Technology, \\ Shanghai 200237, China \\ ajszhang.vita@hotmail.com, ${ }^{\mathrm{a}}$ kanhengzhou@gmail.com, ${ }^{\mathrm{c}}$ jrzheng@126.com
}

Keywords: Evaluation Algorithm, Evaluation Model, Operator Training, Dynamic Programming.

\begin{abstract}
An intelligent evaluation algorithm based on dynamic programming has been proposed because of the high flexibility of operation sequence in the operator training examination which causes the problems in finding the most appropriate answer difficultly. The evaluation model and the maximum matching algorithm of the path has been proposed, which can realize the maximum matching of the operation path and point out the inhibitive operations.
\end{abstract}

\section{Introduction}

With the development of computer science and information technology, the form of examination has evolved towards paperless, intelligent direction, which leads to the emergence of many kinds intelligent examination system, such as the remote experimental platform, the virtual-reality training system, the semi-physical simulation training system, etc. However, the operation path of an actual test is random because of a variety of possibility, which leads to impossibility in making the best judgment manually and difficulties in matching the standard operation path with the actual one objectively.

By far, the path-matching determination method carries out an evaluation according to the sequence of operation steps, which can be applied to most practice examinations. It can be divided into leading operations and subsequent operations [1-2], the problem of which is that the subsequent operations will be determined invalid when leading operations are not finished. The MTL (MaxScore-Topology-Link) algorithm [3] has been proposed, but it is unable to identify the inhibitive operations and just has a single evaluation criteria.

An intelligent evaluation algorithm based on operation path has been proposed, which can realize the most appropriate evaluation based on the logical relationship of the actual operations, pointing out the inhibitive operations and carrying out the comprehensive evaluation of the examination.

\section{Establishment of Operation Evaluation Model}

Assumptions and Definitions of the Model. The operation path is a serial combination of a set of operation steps, which is represented by the set of operations $\left\{O_{1}, O_{2}, \cdots, O_{n}, o_{1}, O_{2}, \cdots o_{n}\right\}$. In which $O_{i}$ is defined as the $i_{\text {th }}$ step's necessary operation and is $o_{i}$ the inhibitive one.

An actual operator's task always can be evaluated by several correctly equivalent-operation paths, the set of which is called evaluation path (EP) set. The logical relationships and types of operations are defined as follows according to all kinds of possibilities in practical situations.

Definition of logical relationships of operations:

Operation Step: "step" for short, a specific operation in the actual operation path, which is the smallest element in operation path.

Serial Process: The order of each step in the process is serial and cannot be reversed between them.

Parallel Processes: The order of each step in the process is parallel. So there is no sequence.

Parallel Block: "block" for short, composed by a number of processes, the logical relationship between which is parallel.

Serial Phase: "phase” for short, composed by a number of processes and blocks, the logical relationship between which is serial. 
Taking the real test for example, as shown in figure 1, uppercase letters stand for necessary operations (the essential step for the operator's task) while lowercase letters stand for inhibitive operations (it may cause serious consequences and is not allowed in the operation path). And sibling arrows represent the parallel relationship whereas single arrows represent the serial relationship. There is no order requirement between parallel process $\mathrm{D}, \mathrm{E}$ and independent operations $\mathrm{X}$, Y can be performed repeatedly in the task and is not restricted to the order. So are the parallel blocks. According to permutations and combinations, there are $2 \times 2 \times 2 \times 2=16$ reasonable EPs in this examination question.

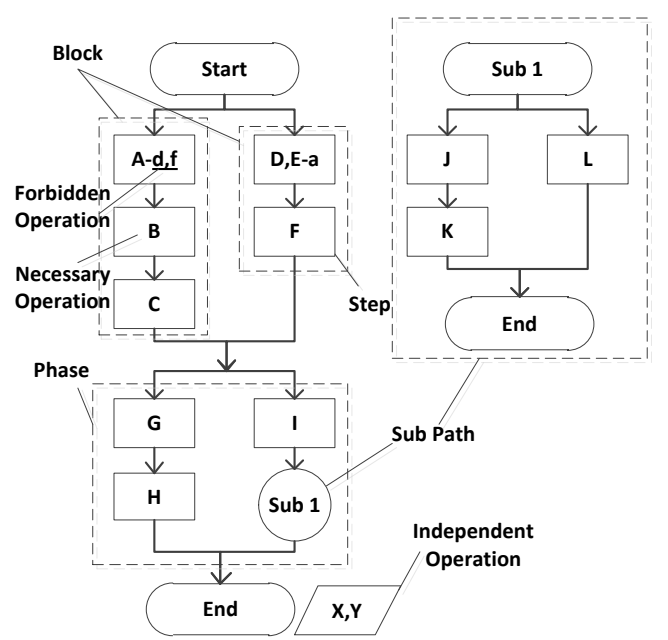

Fig. 1 The example of a real test

Evaluation Index. The evaluation index of the actual operation includes: the operation name, the basic score, the operation time, the number of consecutively correct operations, the operation priority and the system status.

Operation Name: it is the identification of the operation such as $O_{i}$ or $o_{i}$, which is used to distinguish different operations.

Basic Score: the score of the operation $o_{i}$ or $o_{i}$.

Operation Time: it is the time interval between two steps of operator's operations, which is used to determine the proficiency of the operator.

The number of consecutively correct operations: the number of correct operations, whose adjacent operations are also correct, is used to determine the mastery of the operator.

Operation priority: it is used to determine the relative importance of the operation to the whole operating process. The higher priority the operation has, the more important it is. Omitting the high-priority operation or (carrying out it in) incorrect operation time will affect the result of the whole task, and may even cause a serious accident. Therefore, if the operator makes mistakes on high-priority operations, he would lose more marks, such as losing the marks of the whole block or phase, than that on the basic ones. Combined with the index of blocks and phases, it can be realized that if the previous operation is unfinished, the following ones will be determined as missing.

\section{The Operation-Path-Maximum-Matching (OPMM) Algorithm}

The core idea of OPMM proposed in this paper is to find an evaluative sub-path, which has the most operation steps, form the actual operation path. This sub-path needs to meet the following two conditions, namely, that (1) the logical relationship of the sub-path must be the same as that of the EP; (2) the sub-path contains the most operation steps.

In order to automatically evaluate the operator's actual operations according to the evaluation model, the system will read the actual operation path after the operator finishing the task to find the maximum-matching pair (MMP) according to the OPMM. Its implementation steps are as follows:

Marking the status of all the steps in both actual path and EP as "Unmatched". Marking the status of the steps in the EP, which is not contained in the real path, as "Missing". Marking the status of the steps in the real path, which is not contained in the EP, as "unrelated". 
Assuming that the step numbers of "Unmatched" in the EP and actual path are $m$ and $n$. The possibly max step number $(u)$ of the matched steps is the smaller one between $m$ and $n$, marked as $u=\operatorname{Min}(m, n)$.

Obtaining $m^{\prime}$ EPs by fully permuting the $m$ "Unmatched" steps according to various logical relationships such as series between steps, series between phases, parallel between blocks and parallel between the operations in steps.

Picking $u$ steps in order from the $n$ "Unmatched" steps in the actual operations as sub-path. Then there will be $C_{n}^{u}$ sub-paths.

Comparing the $j_{\text {th }}$ step in the $i_{\text {th }}$ actual sub-path form $C_{n}^{u}$ ones with the $l_{\text {th }}$ step in the $k_{\text {th }}$ EP. (The initial value of $i, j, k, l$ is 1 .)

If the result of the comparison is true, which means the actual operation is matched with the excepted one, the actual operation will be considered as the correct operation, then the OPMM will move to the next step, $j=j+1$. If $j$ is larger than $u$, which means that each step in the actual operation sub-path can find the correspondingly matched step in the EP, the two paths will be the target solution (the MMP), and the search will end and go to STEP 8. Otherwise, if $j$ is smaller than $i$ or equal to $i$, the OPMM will go back to STEP 5 and keep searching.

If the result of the comparison is false, which means the actual operation is not matched with the excepted one but it still may be matched with one of the following steps in the EP, the OPMM will move to the next step and keep searching, $l=l+1$. There are two conditions that (1) if $l$ is larger than $m^{\prime}$, which means that the matched operation cannot be found in the $k_{\mathrm{th}} \mathrm{EP}$ after comparing all the steps, the OPMM will keep searching in the next EP $(k=k+1)$. When $k$ is larger than $m^{\prime}$, which means that the matched operation still cannot be found after searching all possible paths, the only way to solve this problem is to reduce the expected number of matched steps $(u=u-1)$. And If $u=0$, which means that MMP cannot be found, the OPMM will stop searching and go to the next STEP , otherwise, it will go back to STEP 4. (2) If $l$ is smaller than or equal to $m$, the OPMM will go back to STEP 5.

Marking the status of the matched steps in the MMP as "Correct".

Determining whether there exist inhibitive operations among all the "Unmatched" steps in the actual operation path or not. The process begins with locating the first "Correct" step, after all "Unmatched" ones, in the actual operation path. And then searching backwards from the location of the first "Correct" step for the "Inhibitive" operations by comparing all "Unmatched"operations in the actual operation path with the "Inhibitive" ones in the EP. If there exists a pair of matched operations, then marking both two steps as "Inhibitive", otherwise, they will be marked as "Irrelevant". Then determine the next "Unmatched" step.

Marking the status of all the rest "Unmatched" steps in the EP as "Missing” and marking the status of all the rest "Unmatched" steps in the actual operation path as "irrelevant".

Putting the matching status and evaluation index of the steps in the EP into the evaluation algorithm to calculate the corresponding score.

\section{The example of a real test}

Taking the real test for example, as shown in figure 1, the basic score of each operation is set to 5 points and the operation time of each operation is set to 5 seconds. What is worth mentioning is that the operation priority of operation d is set to "Block No Points", which means that if the necessary operation in the block is omitted or the inhibitive operation is performed, then the score of the whole parent block will be deducted. Assuming that the actual operation path of the operator is Z,D,B,C,E,D,X,J,L,H,G, then the most reasonable EP is A,d,f,B,C,E,a,D,a,F,I,J,K,L,G,H (XY) according to the OPMM. Limited by paper length, 4 typical EPs have been chosen from all 16 EPs to verify the OPMM, as shown in table 1. 
The EP 1 in table 1 is based on the OPMM, while the EP 2 is according to the MTL. The EPs 3 and 4 here are for reference. Due to the operation priority, the deduction of operation $\mathrm{d}$ is the score of the whole block (15 points).

Compared with the OPMM, the MTL gave a higher score, but it didn't did not recognize the inhibitive operations, which is not strict enough in evaluating the operating purpose of the operators and may cause serious accidents easily in practice. While the OPMM figured out the inhibitive operations and evaluated the operating purpose more reasonably. Compared with the OPMM, one more missing step was determined in the EP 3 because the changeable order of the parallel process D and E had not been taken into account. And so was the EP 4.

Table 1 the Evaluation on the Actual Operation Paths

\begin{tabular}{|c|c|c|c|c|c|c|c|c|c|c|c|}
\hline \multicolumn{3}{|c|}{ EP 1 (OPMM) } & \multicolumn{3}{|c|}{ EP 2 (MTL) } & \multicolumn{3}{|c|}{ EP 3} & \multicolumn{3}{|c|}{ EP 4} \\
\hline Ops & Mark & Score & Ops & Mark & Score & Ops & Mark & Score & Ops & Mark & Score \\
\hline A & Missing & \multirow{5}{*}{-15.0} & $\mathrm{D}$ & Correct & & A & Missing & \multirow{5}{*}{-15.0} & A & Missing & \multirow{5}{*}{-15.0} \\
\hline $\mathrm{d}$ & Inhibitive & & $\mathrm{a}$ & & & $\mathrm{d}$ & Inhibitive & & $\mathrm{d}$ & Inhibitive & \\
\hline $\mathrm{f}$ & & & $\mathrm{E}$ & Missing & -5.0 & $\mathrm{f}$ & & & $\mathrm{f}$ & & \\
\hline B & Correct & & $\mathrm{a}$ & & & B & Correct & & B & Correct & \\
\hline $\mathrm{C}$ & Correct & & $\mathrm{F}$ & Missing & -5.0 & $\mathrm{C}$ & Correct & & $\mathrm{C}$ & Correct & \\
\hline $\mathrm{E}$ & Correct & & A & Missing & -5.0 & $\mathrm{D}$ & Missing & -5.0 & $E$ & Correct & \\
\hline $\mathrm{a}$ & & & $\mathrm{d}$ & & & $\mathrm{a}$ & & & $\mathrm{a}$ & & \\
\hline $\mathrm{D}$ & Correct & & $\mathrm{f}$ & & & $\mathrm{E}$ & Correct & & $\mathrm{D}$ & Correct & \\
\hline $\mathrm{a}$ & & & B & Correct & & $\mathrm{a}$ & & & $\mathrm{a}$ & & \\
\hline $\mathrm{F}$ & Missing & -5.0 & $\mathrm{C}$ & Correct & & $\mathrm{F}$ & Missing & -5.0 & $\mathrm{~F}$ & Missing & -5.0 \\
\hline $\mathrm{I}$ & Missing & -5.0 & $\mathrm{I}$ & Missing & -5.0 & I & Missing & -5.0 & G & Missing & -5.0 \\
\hline $\mathrm{J}$ & Correct & & $\mathrm{J}$ & Correct & & $\mathrm{J}$ & Correct & & $\mathrm{H}$ & Missing & -5.0 \\
\hline K & Missing & -5.0 & K & Missing & -5.0 & K & Missing & -5.0 & I & Missing & -5.0 \\
\hline $\mathrm{L}$ & Correct & & $\mathrm{L}$ & Correct & & $\mathrm{L}$ & Correct & & $\mathrm{J}$ & Correct & \\
\hline $\mathrm{G}$ & Missing & -5.0 & G & Missing & -5.0 & G & Missing & -5.0 & $\bar{K}$ & Missing & -5.0 \\
\hline $\mathrm{H}$ & Correct & & $\mathrm{H}$ & Correct & & $\overline{\mathrm{H}}$ & Correct & & $\mathrm{L}$ & Correct & \\
\hline
\end{tabular}

In summary, not only can the EP picked by the OPMM evaluate the operating purpose of the operators reasonably but also it can figure out the inhibitive operations strictly, which ensures the operators can obtain the highest score based on the operational safety.

\section{Conclusions}

An intelligent evaluation method based on dynamic programming has been proposed to fill the blank that there is no common method for evaluating the operation path in current operator training system. The method can not only realize the maximum matching of the operation path but also can point out the inhibitive operations, and gives the comprehensive evaluation of the examination automatically. Because of the excellent robustness, the method can be applied to various types of operator training system.

\section{References}

[1] Lee S. Jeong I. Il M., Development of evaluation algorithms for operator training system, J. Computers and Chemical Engineering. 2 (2000) p.1517-1522.

[2] Teng Liming, Wu Jia, etc., Evaluating and scoring system for experimental operations based on object-oriented Methods, J. Research and Exploration in Laboratory. 23, 2 (2004) p.63-65.

[3] Zhao Wei, Yang Xu, etc., Design and implementation of examination evaluation system for physics experiment based on the operation path, J. Electronics Design \& Application. 39, 6 (2012) p.56-59. 